\title{
GeOgRaPhic Distribution OF RYEgRASS RESISTENT TO THE Clethodim Herbicide in Rio Grande do Sul ${ }^{1}$
}

\author{
Distribuição Geográfica de Azevém Resistente ao Herbicida Clethodim no Rio Grande do Sul
}

VARGAS, L. ${ }^{2}$, SCHNEIDER, T. ${ }^{3}$, AGOSTINETTO, D. ${ }^{3}$, and BIANCHI, M.A. ${ }^{4}$

\begin{abstract}
Ryegrass is a weed of annual cycle that is present in winter crops, in orchards and vineyards of the South region of Brazil. The species is normally controlled by the glyphosate herbicide, but the continuous use of this product caused the selection of resistant biotypes. The use of ACCase inhibitor herbicides is the main alternative for the control of this species, but it has not been satisfactory in some places, thus causing suspicion of resistance to this action mechanism. Thus, the objective of this paper was to evaluate the occurrence and geographic distribution of ryegrass biotypes that are resistant to the clethodim herbicide in the state of Rio Grande do Sul. For that, seeds of ryegrass plants that survived the application of clethodim were collected in crops from the north region of RS, summing up to a total of 152 samples from 72 cities. The biotypes were submitted to the application of $120 \mathrm{~g}$ i.a. ha ${ }^{-1}$ (maximum registered dose) and $60 \mathrm{~g}$ i.a. ha-1 de clethodim (half the maximum registered dose). According to the results, among the samples of ryegrass seeds collected, there were no biotypes resistant to the clethodim herbicide when the maximum registered dose was applied and in the stage of three to four leaves. However, there were biotypes with lower susceptibility that survived half the maximum registered dose.
\end{abstract}

Keywords: Lolium multiflorum, resistance, chemical control, acetyl coenzyme A carboxylase.

\begin{abstract}
RESUMO - O azevém é uma planta daninha de ciclo anual presente em lavouras de inverno, em pomares e vinhedos da região Sul do Brasil. A espécie normalmente é controlada pelo herbicida glyphosate, porém o uso continuado desse produto selecionou biótipos resistentes. O uso de herbicidas inibidores da ACCase é a principal alternativa para o controle dessa espécie, o qual não tem sido satisfatório em alguns locais, provocando a suspeita da ocorrência de resistência a esse mecanismo de ação. Assim, o objetivo deste trabalho foi avaliar a ocorrência e distribuição geográfica de biótipos de azevém resistente ao herbicida clethodim no Estado do Rio Grande do Sul. Para isso, sementes de plantas de azevém que sobreviveram a aplicações de clethodim foram coletadas em lavouras da região norte do RS, totalizando 152 amostras de 72 municipios. Os biótipos foram submetidos à

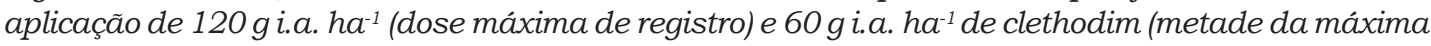
dose de registro). De acordo com os resultados, entre a amostras de sementes de azevém coletadas não foram encontrados biótipos resistentes ao herbicida clethodim quando aplicada a máxima dose de registro e no estádio de três a quatro folhas. No entanto, observaram-se biótipos com menor suscetibilidade, que sobreviveram à metade da máxima dose de registro.
\end{abstract}

Palavras-chave: Lolium multiflorum, resistência, controle químico, acetyl coenzima A carboxilase.

\section{INTRODUCTION}

In tillage system or in orchards, ryegrass control is usually done by applying nonselective herbicides, mostly glyphosate. The intensive use of glyphosate for this end is due to its high efficiency in different vegetative stages of the species, combined with its low cost when compared to the other herbicides (Christoffoleti \& Lópes-Ovejero, 2003). The

1 Recebido para publicação em 7.9.2015 e aprovado em 13.11.2015.

2 Embrapa Trigo, Passo Fundo-RS, Brazil, <leandro.vargas@embrapa.br $>$; ${ }^{3}$ Universidade Federal de Pelotas, Pelotas- RS, Brazil, ${ }^{4}$ CCGL-Tec/Universidade de Cruz Alta, Cruz Alta-RS, Brazil. 
intensive use of an herbicide imposes high selection pressure on the population of plants, resulting in the selection of resistant biotypes pre-existent in the population (Powles \& Yu, 2010).

Resistance is defined as the inherent and inheritable capability of a biotype, within a certain population, of surviving and reproducing after exposure to the registered dose of the herbicide for the control of the species, according to the application criteria (Grazziero et al., 2009). The resistance of weeds to herbicides is an evolution process, and the dynamics and the impact depend on several factors. The factors that are able to determine how the resistance is boosted are the genetic diversity of the population, the existence of genes resistant to a specific herbicide and operational factors (Powles \& Yu, 2010).

As an alternative for the control of ryegrass populations that are resistant to glyphosate, especially in pre-sowing of maize and wheat, the herbicides used are the ones that inhibit the ACCase enzyme. The ACCase inhibitor herbicides are applied in the weed postemergence period; they control species of poaceae and are selective for magnoliopsida crops (Vidal \& Merotto Jr., 2001). The repeated use of ACCase inhibitor herbicides resulted in the selection of biotypes resistant to this action mechanism in Brazil. The bioecological characteristics of the ryegrass, such as genetic variability, allogamy and elevated production of seeds, associated to inappropriate handling strategies, make the selection of resistant biotypes predictable and worrisome (Christoffoleti \& López-Ovejero, 2003).

Facing a scenario of elevated resistance of weeds to herbicides, it is crucial to have measures that recommend the handling of the resistance, and it is important to monitor crops for the identification of the resistance focus and the elimination of suspected plants (Lazaroto et al., 2008). After resistance is identified in the field, the adoption of practices such as crop rotation, mixture of herbicides, localized applications and association of control methods reduces the impact of the problem (Hugh, 2006).

Knowing the resistant species and the extension of the infested area provides insight on the severity of the problem, making it possible to determine the resistance handling and making the process of selection of resistant biotypes slower, helping in the reactive decision making process in the control of these populations (Owen et al., 2014). In addition, mapping the resistance enables the establishment of the resistance costs, the definition of public policies and the directed technical assistance.

In Brazil, there is no information gathering on the area infested with ACCase inhibitor herbicide-resistant ryegrass. Gathering information of ryegrass populations that are resistant to ACCase inhibitor herbicides is a tool that will enable the identification of the places where this resistance occurs and the determination of specific handling strategies for each region, according to its characteristics. With this information, the decision making process and the adoption of prevention and resistance control strategies become possible, recovering the viability of cultivation in these areas. The objective of this paper was to evaluate the geographic distribution of ryegrass biotypes that are resistant to clethodim in the state of Rio Grande do Sul.

\section{MATERIAL AND METHODS}

To perform this paper, we collected seeds of ryegrass plants that survived the application of ACCase inhibitor herbicides in crops of the north region of Rio Grande do Sul. The seeds that composed the samples came from a plant, and all the collection points were identified with geodetic coordinates, through the use of the Global Positioning System (GPS).

The harvest of seeds happened between the months of September and November 2013, in properties located in the following cities: Água Santa (3), Alto Alegre (2), Augusto Pestana (2), Barra Funda (2), Barracão (4), Boa Vista do Cadeado, Boa Vista do Incra (3), Bom Progresso, Campo Novo, Capão Bonito do Sul, Carazinho (3), Caseiros (2), Ciríaco (2), Colorado, Condor, Coqueiros do Sul (5), Coronel Bicaco, Coxilha (2), Crissiumal (4), Cruz Alta (3), David Canabarro (2), Ernestina, Erval Seco (3), Espumoso (2), Frederico Westphalen, Gentil, Horizontina (2), Humaitá (2), Ibiaçá (2), Ibiraiaras (2), Independência (2), Joia (3), 
Julio de Castilhos (3), Lagoa Vermelha (3), Machadinho (4), Mato Castelhano, Maximiliano de Almeida, Muitos Capões (2), Nova Candelária, Novo Barreiro, Paim Filho (2), Palmeira das Missões (2), Panambi (4), Passo Fundo, Pejuçara (3), Saldanha Marinho (2), Salto do Jacuí, Sananduva (3), Santa Rosa, Santa Bárbara do Sul (3), Santa Cecília do Sul, Santo Augusto, Santo Cristo (2), São João da Urtiga, São José do Inhacorá, São José do Ouro (2), São Miguel das Missões, Seberi (2), Selbach, Tapejara (2), Tapera, Taquaruçu do Sul (2), Tenente Portela (2), Tio Hugo (2), Três de Maio (4), Três Passos (2), Tucunduva (3), Tupanciretã (2), Tuparendi, Vacaria (2), Victor Graeff, Vila Lângaro (4) and Vista Alegre (Figure 1), summing up 152 samples of seeds harvested in 74 cities.

After the harvest, the seeds were cleaned, identified and stored until when they would be used in experiments. The experiment, carried out to confirm resistance, was done between October and December 2013, in a greenhouse, in a completely randomized design, and the treatments were arranged in a factorial scheme, in which the A factor was composed of biotypes of different places of harvest and B had two doses of the clethodim herbicide $(60$ and $120 \mathrm{~g}$ i.a. $\mathrm{ha}^{-1}$ ), representing half the



Source: Adapted from Geolivre - Mapas temáticos RS, 2011.

Figure 1 - Geographic location of the cities where there was harvest of seeds of ryegrass plants suspected of being resistant to the clethodim herbicide in the state of Rio Grande do Sul. maximum registered dose and the maximum registered dose, respectively, for the control of ryegrass in pre-sowing of maize and wheat (AGROFIT, 2015). A control treatment without herbicide application was also added. The experimental units were composed of plastic vases with volumetric capacity of $500 \mathrm{~mL}$, containing commercial substrate, being composed of five plants per vase.

When the plants reached a vegetative state of three to four leaves, the herbicide was applied, using the backpack sprayer, pressured at $\mathrm{CO}_{2}$, calibrated to provide application volume of $120 \mathrm{~L} \mathrm{ha}^{-1}$ of herbicide spray, equipped with spray nozzles in the form of a fan 110.015. The Lanzar ${ }^{\circledR}$ adjuvant was also used in the spray in the dose of $0,5 \% \mathrm{v} / \mathrm{v}$.

The variable evaluated was visual control at 28 days after the application of treatments (DAT), and the biotypes were identified, according to the response to the herbicides, as susceptible or resistant, adopting a binary scale in which zero $(0)$ represented the death of the plants and one (1) represented survival. The data obtained was analyzed by descriptive statistics, seeking to establish relations between the distributions of cases of ryegrass suspected to be resistant to the ACCase enzyme inhibitors.

\section{RESULTS AND DISCUSSION}

The data analysis revealed that all the 152 harvested biotypes were controlled with a doses of $120 \mathrm{~g}$ i.a. ha ${ }^{-1}$ clethodim, proving the nonoccurrence of resistance to this herbicide (Table 1). However, 14 biotypes survived the application of half the maximum registered dose (60 g i.a. ha-1) for the control of ryegrass, showing sensitivity difference. Similar results were observed in biotypes sensitivity studies of Euphorbia heterophylla and Eleusine spp. with suspicion of resistance to the glyphosate herbicide, originated from soy crops RR from Rio Grande do Sul - RS (Ulguim, 2012; Vargas et al., 2013).

The use of the maximum registered dose of the herbicide is due to the fact that a plant can only be classified as resistant to the herbicide if it survives and reproduces after exposure to the registered dose of the product for the control of the species, according to the 
Table 1 - Localization and response $(0=$ death, 1 = survival $)$ of biotypes of Lolium multiflorum in function of the application of 60 and $120 \mathrm{~g} \mathrm{ha}^{-1}$ of clethodim visually evaluated at 28 days after the treatment (DAT)

\begin{tabular}{|c|c|c|c|c|c|}
\hline \multirow{2}{*}{ Biotype } & \multirow{2}{*}{ City } & \multicolumn{2}{|c|}{ Coordinates } & \multicolumn{2}{|c|}{ Responses to Clethodim } \\
\hline & & Latitude & Longitude & $60 \mathrm{~g}$ & $120 \mathrm{~g}$ \\
\hline AGS 01 & Água Santa & $28^{\circ} 17^{\prime} 38^{\prime \prime}$ & $51^{\circ} 58^{\prime} 34^{\prime \prime}$ & 0 & 0 \\
\hline AGS 02 & Água Santa & $28^{\circ} 15^{\prime} 11^{\prime \prime}$ & $51^{\circ} 58^{\prime} 50^{\prime \prime}$ & 1 & 0 \\
\hline AGS 03 & Água Santa & $28^{\circ} 14^{\prime} 38^{\prime \prime}$ & $51^{\circ} 58^{\prime} 57^{\prime}$, & 1 & 0 \\
\hline ALA 01 & Alto Alegre & $28^{\circ} 46^{\prime} 34^{\prime \prime}$ & $52^{\circ} 56^{\prime} 15^{\prime \prime}$ & 0 & 0 \\
\hline ALA 02 & Alto Alegre & $28^{\circ} 46^{\prime} 45^{\prime \prime}$ & $52^{\circ} 59^{\prime} 06^{\prime \prime}$ & 0 & 0 \\
\hline APE 01 & Augusto Pestana & $28^{\circ} 31^{\prime} 43^{\prime \prime}$ & $54^{\circ} 01^{\prime} 11^{\prime \prime}$ & 0 & 0 \\
\hline APE 02 & Augusto Pestana & $28^{\circ} 33^{\prime} 04^{\prime \prime}$ & $54^{\circ} 03^{\prime} 51^{\prime \prime}$ & 0 & 0 \\
\hline BAF 01 & Barra Funda & $28^{\circ} 55^{\prime} 55^{\prime \prime}$ & $53^{\circ} 03^{\prime} 27^{\prime \prime}$ & 0 & 0 \\
\hline BAF 02 & Barra Funda & $27^{\circ} 55^{\prime} 59^{\prime \prime}$ & $53^{\circ} 00^{\prime} 30^{\prime \prime}$ & 0 & 0 \\
\hline BAR 01 & Barracão & $27^{\circ} 48^{\prime} 60^{\prime \prime}$ & $51^{\circ} 26^{\prime} 42^{\prime}$ & 0 & 0 \\
\hline BAR 02 & Barracão & $27^{\circ} 39^{\prime} 51^{\prime \prime}$ & $51^{\circ} 32^{\prime}{ }^{\prime} 55^{\prime}$ & 0 & 0 \\
\hline BAR 03 & Barracão & $27^{\circ} 35^{\prime} 55^{\prime \prime}$ & $51^{\circ} 38^{\prime} 25^{\prime \prime}$ & 0 & 0 \\
\hline BAR 04 & Barracão & $27^{\circ} 41^{\prime} 04^{\prime \prime}$ & $51^{\circ} 30^{\prime} 53^{\prime \prime}$ & 0 & 0 \\
\hline BVC 01 & Boa Vista do Cadeado & $28^{\circ} 32^{\prime} 45^{\prime \prime}$ & $53^{\circ} 47^{\prime} 40^{\prime \prime}$ & 0 & 0 \\
\hline BVI 01 & Boa Vista do Incra & $28^{\circ} 56^{\prime} 40^{\prime \prime}$ & $53^{\circ} 24^{\prime} 59^{\prime \prime}$ & 0 & 0 \\
\hline BVI 02 & Boa Vista do Incra & $28^{\circ} 56^{\prime} 45^{\prime \prime}$ & $53^{\circ} 24^{\prime} 49^{\prime \prime}$ & 0 & 0 \\
\hline BVI 03 & Boa Vista do Incra & $28^{\circ} 52^{\prime} 38^{\prime \prime}$ & $53^{\circ} 27^{\prime} 51^{\prime \prime}$ & 0 & 0 \\
\hline BOP & Bom Progresso & $27^{\circ} 34^{\prime} 25^{\prime \prime}$ & $53^{\circ} 51^{\prime} 58^{\prime \prime}$ & 0 & 0 \\
\hline CAN & Campo Novo & $27^{\circ} 40^{\prime} 48^{\prime \prime}$ & $53^{\circ} 50^{\prime} 31^{\prime \prime}$ & 0 & 0 \\
\hline CBS & Capão Bonito do Sul & $28^{\circ} 18^{\prime} 60^{\prime \prime}$ & $51^{\circ} 21^{\prime} 09^{\prime}$ & 0 & 0 \\
\hline CAR 01 & Carazinho & $28^{\circ} 20^{\prime} 09^{\prime \prime}$ & $52^{\circ} 55^{\prime} 34^{\prime \prime}$ & 0 & 0 \\
\hline CAR 02 & Carazinho & $28^{\circ} 20^{\prime} 36^{\prime \prime}$ & $52^{\circ} 51^{\prime} 19^{\prime \prime}$ & 0 & 0 \\
\hline CAR 03 & Carazinho & $28^{\circ} 20^{\prime} 34^{\prime \prime}$ & $52^{\circ} 54^{\prime} 39^{\prime \prime}$ & 0 & 0 \\
\hline CAS 01 & Caseiros & $28^{\circ} 13^{\prime} 30^{\prime \prime}$ & $51^{\circ} 38^{\prime} 27^{\prime \prime}$ & 0 & 0 \\
\hline CAS 02 & Caseiros & $28^{\circ} 16^{\prime} 44^{\prime \prime}$ & $51^{\circ} 40^{\prime \prime} 44^{\prime \prime}$ & 0 & 0 \\
\hline CIR 01 & Ciríaco & $28^{\circ} 18^{\prime} 26^{\prime \prime}$ & $51^{\circ} 57^{\prime} 11^{\prime \prime}$ & 0 & 0 \\
\hline CIR 02 & Ciríaco & $28^{\circ} 19^{\prime} 06^{\prime \prime}$ & $51^{\circ} 54^{\prime} 34^{\prime}$ & 0 & 0 \\
\hline COL 01 & Colorado & $28^{\circ} 22^{\prime} 18^{\prime \prime}$ & $52^{\circ} 17^{\prime} 21^{\prime \prime}$ & 0 & 0 \\
\hline CON 01 & Condor & $28^{\circ} 09^{\prime} 49^{\prime \prime}$ & $53^{\circ} 28^{\prime} 05^{\prime \prime}$ & 0 & 0 \\
\hline COQ 1 & Coqueiros do Sul & $28^{\circ} 11^{\prime} 20^{\prime \prime}$ & $52^{\circ} 44^{\prime} 33^{\prime \prime}$ & 0 & 0 \\
\hline COQ 2 & Coqueiros do Sul & $28^{\circ} 11^{\prime} 09^{\prime \prime}$ & $52^{\circ} 44^{\prime} 19^{\prime}$ & 1 & 0 \\
\hline COQ 3 & Coqueiros do Sul & $28^{\circ} 11^{\prime} 23^{\prime \prime}$ & $52^{\circ} 44^{\prime} 38^{\prime \prime}$ & 0 & 0 \\
\hline COQ 4 & Coqueiros do Sul & $28^{\circ} 11^{\prime} 37^{\prime \prime}$ & $52^{\circ} 44^{\prime} 23^{\prime \prime}$ & 0 & 0 \\
\hline COQ 5 & Coqueiros do Sul & $28^{\circ} 11^{\prime} 33^{\prime \prime}$ & $52^{\circ} 44^{\prime} 17^{\prime \prime}$ & 1 & 0 \\
\hline $\mathrm{COB}$ & Coronel Bicaco & $27^{\circ} 46^{\prime} 13^{\prime \prime}$ & $53^{\circ} 34^{\prime} 37^{\prime \prime}$ & 0 & 0 \\
\hline COX 01 & Coxilha & $28^{\circ} 07^{\prime} 01^{\prime \prime}$ & $52^{\circ} 15^{\prime} 51^{\prime \prime}$ & 0 & 0 \\
\hline COX 02 & Coxilha & $28^{\circ} 10^{\prime} 06^{\prime \prime}$ & $52^{\circ} 18^{\prime} 51^{\prime \prime}$ & 0 & 0 \\
\hline CRI 01 & Crissiumal & $27^{\circ} 30^{\prime} 55^{\prime}$ & $54^{\circ} 08^{\prime} 13^{\prime \prime}$ & 0 & 0 \\
\hline CRI 02 & Crissiumal & $27^{\circ} 32^{\prime} 37^{\prime \prime}$ & $54^{\circ} 01^{\prime} 18^{\prime \prime}$ & 0 & 0 \\
\hline CRI 03 & Crissiumal & $27^{\circ} 33^{\prime} 06^{\prime \prime}$ & $54^{\circ} 15^{\prime} 34^{\prime \prime}$ & 0 & 0 \\
\hline CRI 04 & Crissiumal & $27^{\circ} 30^{\prime} 47^{\prime \prime}$ & $54^{\circ} 12^{\prime} 12^{\prime \prime}$ & 0 & 0 \\
\hline CRA 01 & Cruz Alta & $28^{\circ} 51^{\prime} 52^{\prime \prime}$ & $53^{\circ} 36^{\prime} 44^{\prime \prime}$ & 0 & 0 \\
\hline CRA 02 & Cruz Alta & $28^{\circ} 47^{\prime} 26^{\prime \prime}$ & $53^{\circ} 36^{\prime} 16^{\prime \prime}$ & 0 & 0 \\
\hline CRA 03 & Cruz Alta & $28^{\circ} 42^{\prime} 47^{\prime \prime}$ & $53^{\circ} 35^{\prime} 07^{\prime \prime}$ & 0 & 0 \\
\hline DAC 01 & David Canabarro & $28^{\circ} 23^{\prime} 53^{\prime \prime}$ & $51^{\circ} 48^{\prime} 02^{\prime \prime}$ & 0 & 0 \\
\hline DAC 02 & David Canabarro & $28^{\circ} 22^{\prime} 57^{\prime \prime}$ & $51^{\circ} 51^{\prime} 11^{\prime \prime}$ & 1 & 0 \\
\hline ERN 01 & Ernestina & $27^{\circ} 33^{\prime} 19^{\prime \prime}$ & $52^{\circ} 30^{\prime} 35^{\prime \prime}$ & 0 & 0 \\
\hline ERS 01 & Erval Seco & $27^{\circ} 31^{\prime} 16^{\prime \prime}$ & $53^{\circ} 28^{\prime} 31^{\prime \prime}$ & 1 & 0 \\
\hline ERS 02 & Erval Seco & $27^{\circ} 31^{\prime} 24^{\prime \prime}$ & $53^{\circ} 28^{\prime} 17^{\prime}$ & 0 & 0 \\
\hline ERS 03 & Erval Seco & $27^{\circ} 31^{\prime} 57^{\prime \prime}$ & $53^{\circ} 28^{\prime} 39^{\prime \prime}$ & 0 & 0 \\
\hline ESP 01 & Espumoso & $28^{\circ} 49^{\prime} 39^{\prime \prime}$ & $52^{\circ} 54^{\prime} 08^{\prime \prime}$ & 0 & 0 \\
\hline ESP 02 & Espumoso & $28^{\circ} 52^{\prime} 18^{\prime \prime}$ & $52^{\circ} 43^{\prime} 19^{\prime \prime}$ & 1 & 0 \\
\hline
\end{tabular}


Table 1, cont.

\begin{tabular}{|c|c|c|c|c|c|}
\hline \multirow{2}{*}{ Biotype } & \multirow{2}{*}{ City } & \multicolumn{2}{|c|}{ Coordinates } & \multicolumn{2}{|c|}{ Responses to Clethodim } \\
\hline & & Latitude & Longitude & $60 \mathrm{~g}$ & $120 \mathrm{~g}$ \\
\hline FRW 01 & Frederico Westphalen & $27^{\circ} 24^{\prime} 12^{\prime \prime}$ & $53^{\circ} 24^{\prime} 41^{\prime \prime}$ & 0 & 0 \\
\hline GEN 01 & Gentil & $28^{\circ} 18^{\prime} 57^{\prime \prime}$ & $51^{\circ} 02^{\prime} 06^{\prime \prime}$ & 0 & 0 \\
\hline HOR 01 & Horizontina & $27^{\circ} 36^{\prime} 23^{\prime \prime}$ & $54^{\circ} 21^{\prime} 30^{\prime \prime}$ & 0 & 0 \\
\hline HOR 02 & Horizontina & $27^{\circ} 35^{\prime} 09^{\prime \prime}$ & $54^{\circ} 20^{\prime} 57^{\prime \prime}$ & 0 & 0 \\
\hline HUM 01 & Humaitá & $27^{\circ} 33^{\prime} 12^{\prime \prime}$ & $53^{\circ} 55^{\prime} 39^{\prime \prime}$ & 0 & 0 \\
\hline HUM 02 & Humaitá & $27^{\circ} 34^{\prime} 19^{\prime \prime}$ & $53^{\circ} 55^{\prime} 54^{\prime \prime}$ & 0 & 0 \\
\hline IBI 01 & Ibiaçá & $28^{\circ} 03^{\prime} 37^{\prime \prime}$ & $51^{\circ} 49^{\prime} 44^{\prime \prime}$ & 0 & 0 \\
\hline IBI 02 & Ibiaçá & $28^{\circ} 03^{\prime} 47^{\prime \prime}$ & $51^{\circ} 50^{\prime} 43^{\prime \prime}$ & 0 & 0 \\
\hline IBR 01 & Ibiraiaras & $28^{\circ} 20^{\prime} 47^{\prime \prime}$ & $51^{\circ} 38^{\prime} 49^{\prime \prime}$ & 1 & 0 \\
\hline IBR 02 & Ibiraiaras & $28^{\circ} 20^{\prime} 34^{\prime \prime}$ & $51^{\circ} 38^{\prime} 45^{\prime \prime}$ & 0 & 0 \\
\hline IND 01 & Independência & $27^{\circ} 52^{\prime} 54^{\prime \prime}$ & $54^{\circ} 11^{\prime} 49^{\prime \prime}$ & 0 & 0 \\
\hline IND 02 & Independência & $27^{\circ} 52^{\prime} 28^{\prime \prime}$ & $54^{\circ} 11^{\prime} 52^{\prime \prime}$ & 0 & 0 \\
\hline JOI 01 & Joia & $28^{\circ} 44^{\prime} 18^{\prime \prime}$ & $54^{\circ} 10^{\prime} 07^{\prime \prime}$ & 0 & 0 \\
\hline JOI 02 & Joia & $28^{\circ} 43^{\prime} 33^{\prime \prime}$ & $54^{\circ} 09^{\prime} 56^{\prime \prime}$ & 0 & 0 \\
\hline JOI 03 & Joia & $28^{\circ} 44^{\prime} 14^{\prime \prime}$ & $54^{\circ} 10^{\prime} 06^{\prime \prime}$ & 0 & 0 \\
\hline JCA 01 & Julio de Castilhos & $29^{\circ} 16^{\prime} 01^{\prime \prime}$ & $53^{\circ} 39^{\prime} 19^{\prime \prime}$ & 0 & 0 \\
\hline JCA 02 & Julio de Castilhos & $29^{\circ} 06^{\prime} 56^{\prime \prime}$ & $53^{\circ} 39^{\prime} 57^{\prime \prime}$ & 0 & 0 \\
\hline JCA 03 & Julio de Castilhos & $29^{\circ} 11^{\prime} 02^{\prime \prime}$ & $53^{\circ} 40^{\prime} 40^{\prime \prime}$ & 0 & 0 \\
\hline LAV 01 & Lagoa Vermelha & $28^{\circ} 04^{\prime} 23^{\prime \prime}$ & $51^{\circ} 27^{\prime} 58^{\prime \prime}$ & 0 & 0 \\
\hline LAV 02 & Lagoa Vermelha & $28^{\circ} 17^{\prime} 52^{\prime \prime}$ & $51^{\circ} 27^{\prime} 01^{\prime \prime}$ & 0 & 0 \\
\hline LAV 03 & Lagoa Vermelha & $28^{\circ} 17^{\prime} 31^{\prime \prime}$ & $51^{\circ} 23^{\prime} 48^{\prime \prime}$ & 0 & 0 \\
\hline MAC 01 & Machadinho & $27^{\circ} 35^{\prime} 55^{\prime}$, & $51^{\circ} 38^{\prime} 25^{\prime}$ & 0 & 0 \\
\hline MAC 02 & Machadinho & $27^{\circ} 39^{\prime} 37^{\prime \prime}$ & $51^{\circ} 33^{\prime} 53^{\prime \prime}$ & 0 & 0 \\
\hline MAC 03 & Machadinho & $27^{\circ} 34^{\prime} 18^{\prime \prime}$ & $51^{\circ} 40^{\prime} 38^{\prime \prime}$ & 0 & 0 \\
\hline MAC 04 & Machadinho & $27^{\circ} 36^{\prime} 46^{\prime \prime}$ & $51^{\circ} 42^{\prime} 18^{\prime \prime}$ & 0 & 0 \\
\hline MCA 01 & Mato Castelhano & $28^{\circ} 18^{\prime} 53^{\prime \prime}$ & $51^{\circ} 08^{\prime} 35^{\prime \prime}$ & 0 & 0 \\
\hline MAX 01 & Maximiliano de Almeida & $27^{\circ} 36^{\prime} 58^{\prime \prime}$ & $51^{\circ} 47^{\prime} 35^{\prime \prime}$ & 0 & 0 \\
\hline MUC 01 & Muitos Capões & $28^{\circ} 19^{\prime} 32^{\prime \prime}$ & $51^{\circ} 15^{\prime} 04^{\prime \prime}$ & 0 & 0 \\
\hline MUC 02 & Muitos Capões & $28^{\circ} 19^{\prime} 34^{\prime \prime}$ & $51^{\circ} 14^{\prime} 10^{\prime \prime}$ & 0 & 0 \\
\hline NCA 01 & Nova Candelária & $27^{\circ} 38^{\prime} 06^{\prime \prime}$ & $54^{\circ} 08^{\prime} 17^{\prime}$ & 0 & 0 \\
\hline NBA 01 & Novo Barreiro & $27^{\circ} 55^{\prime} 07^{\prime \prime}$ & $53^{\circ} 05^{\prime} 11^{\prime \prime}$ & 0 & 0 \\
\hline PAF 01 & Paim Filho & $27^{\circ} 43^{\prime} 30^{\prime \prime}$ & $51^{\circ} 44^{\prime} 27^{\prime \prime}$ & 0 & 0 \\
\hline PAF 02 & Paim Filho & $27^{\circ} 41^{\prime} 48^{\prime \prime}$ & $51^{\circ} 47^{\prime} 53^{\prime}$ & 0 & 0 \\
\hline PAM 01 & Palmeira das Missões & $27^{\circ} 53^{\prime} 53^{\prime \prime}$ & $53^{\circ} 15^{\prime} 45^{\prime \prime}$ & 0 & 0 \\
\hline PAM 02 & Palmeira das Missões & $27^{\circ} 45^{\prime} 51^{\prime \prime}$ & $53^{\circ} 30^{\prime} 06^{\prime \prime}$ & 0 & 0 \\
\hline PAN 01 & Panambi & $28^{\circ} 20^{\prime} 31^{\prime \prime}$ & $53^{\circ} 3360^{\prime \prime}$ & 1 & 0 \\
\hline PAN 02 & Panambi & $28^{\circ} 15^{\prime} 36^{\prime \prime}$ & $53^{\circ} 28^{\prime} 18^{\prime \prime}$ & 0 & 0 \\
\hline PAN 03 & Panambi & $28^{\circ} 21^{\prime} 50^{\prime \prime}$ & $53^{\circ} 27^{\prime} 39^{\prime \prime}$ & 0 & 0 \\
\hline PAN 04 & Panambi & $28^{\circ} 21^{\prime} 03^{\prime \prime}$ & $53^{\circ} 27^{\prime} 39^{\prime \prime}$ & 0 & 0 \\
\hline PAS 01 & Passo Fundo & $28^{\circ} 14^{\prime} 13^{\prime \prime}$ & $52^{\circ} 34^{\prime} 15^{\prime}$ & 0 & 0 \\
\hline PEJ 01 & Pejuçara & $28^{\circ} 19^{\prime} 30^{\prime \prime}$ & $53^{\circ} 39^{\prime} 52^{\prime \prime}$ & 0 & 0 \\
\hline PEJ 02 & Pejuçara & $28^{\circ} 19^{\prime} 27^{\prime \prime}$ & $53^{\circ} 39^{\prime} 48^{\prime \prime}$ & 0 & 0 \\
\hline PEJ 03 & Pejuçara & $28^{\circ} 25^{\prime} 53^{\prime \prime}$ & $53^{\circ} 33^{\prime} 24^{\prime \prime}$ & 1 & 0 \\
\hline SAM 01 & Saldanha Marinho & $28^{\circ} 25^{\prime} 59^{\prime \prime}$ & $53^{\circ} 08^{\prime} 11^{\prime \prime}$ & 0 & 0 \\
\hline SAM 02 & Saldanha Marinho & $28^{\circ} 23^{\prime} 40^{\prime \prime}$ & $53^{\circ} 03^{\prime} 15^{\prime \prime}$ & 0 & 0 \\
\hline SAJ 01 & Salto do Jacuí & $29^{\circ} 03^{\prime} 43^{\prime \prime}$ & $53^{\circ} 0932^{\prime \prime}$ & 0 & 0 \\
\hline SAN 01 & Sananduva & $27^{\circ} 58^{\prime} 20^{\prime \prime}$ & $51^{\circ} 47^{\prime} 35^{\prime \prime}$ & 0 & 0 \\
\hline SAN 02 & Sananduva & $27^{\circ} 53^{\prime} 37^{\prime \prime}$ & $51^{\circ} 49^{\prime} 15^{\prime \prime}$ & 0 & 0 \\
\hline SAN 03 & Sananduva & $27^{\circ} 58^{\prime} 20^{\prime \prime}$ & $51^{\circ} 47^{\prime} 34^{\prime \prime}$ & 0 & 0 \\
\hline SAR 01 & Santa Rosa & $27^{\circ} 50^{\prime} 59^{\prime \prime}$ & $54^{\circ} 3259^{\prime \prime}$ & 0 & 0 \\
\hline SAB 01 & Santa Bárbara do Sul & $28^{\circ} 24^{\prime} 27^{\prime \prime}$ & $53^{\circ} 13^{\prime} 53^{\prime \prime}$ & 0 & 0 \\
\hline SAB 02 & Santa Bárbara do Sul & $28^{\circ} 22^{\prime} 48^{\prime \prime}$ & $53^{\circ} 24^{\prime} 13^{\prime \prime}$ & 0 & 0 \\
\hline SAB 03 & Santa Bárbara do Sul & $28^{\circ} 23^{\prime} 25^{\prime \prime}$ & $53^{\circ} 24^{\prime} 13^{\prime \prime}$ & 0 & 0 \\
\hline
\end{tabular}

To be continued ... 
Table 1, cont.

\begin{tabular}{|c|c|c|c|c|c|}
\hline \multirow{2}{*}{ Biotype } & \multirow{2}{*}{ City } & \multicolumn{2}{|c|}{ Coordinates } & \multicolumn{2}{|c|}{ Responses to Clethodim } \\
\hline & & Latitude & Longitude & $60 \mathrm{~g}$ & $120 \mathrm{~g}$ \\
\hline SAC 01 & Santa Cecília do Sul & $28^{\circ} 15^{\prime} 30^{\prime \prime}$ & $51^{\circ} 59^{\prime} 47^{\prime \prime}$ & $\overline{0}$ & 0 \\
\hline SAG 01 & Santo Augusto & $27^{\circ} 43^{\prime} 08^{\prime \prime}$ & $53^{\circ} 50^{\prime} 58^{\prime \prime}$ & 0 & 0 \\
\hline SCR 01 & Santo Cristo & $27^{\circ} 50^{\prime} 30^{\prime \prime}$ & $54^{\circ} 34^{\prime} 44^{\prime \prime}$ & 0 & 0 \\
\hline SCR 02 & Santo Cristo & $27^{\circ} 50^{\prime} 30^{\prime \prime}$ & $54^{\circ} 35^{\prime} 23^{\prime \prime}$ & 1 & 0 \\
\hline SJU 01 & São João da Urtiga & $27^{\circ} 51^{\prime} 15^{\prime \prime}$ & $51^{\circ} 49^{\prime} 26^{\prime \prime}$ & 0 & 0 \\
\hline SJI 01 & São José do Inhacorá & $2741^{\prime} 37^{\prime \prime}$ & $54^{\circ} 09^{\prime} 12^{\prime \prime}$ & 0 & 0 \\
\hline SJO 01 & São José do Ouro & $27^{\circ} 46^{\prime} 55^{\prime \prime}$ & $51^{\circ} 38^{\prime} 29^{\prime \prime}$ & 0 & 0 \\
\hline SJO 02 & São José do Ouro & $27^{\circ} 43^{\prime} 12^{\prime}$ & $51^{\circ} 32^{\prime} 15^{\prime}$ & 0 & 0 \\
\hline SMM 01 & S. Miguel das Missões & $28^{\circ} 59^{\prime} 31^{\prime \prime}$ & $54^{\circ} 22^{\prime} 35^{\prime \prime}$ & 0 & 0 \\
\hline SEB 01 & Seberi & $27^{\circ} 25^{\prime} 44^{\prime \prime}$ & $53^{\circ} 25^{\prime} 17^{\prime \prime}$ & 0 & 0 \\
\hline SEB 02 & Seberi & $27^{\circ} 25^{\prime} 36^{\prime \prime}$ & $53^{\circ} 25^{\prime} 24^{\prime \prime}$ & 0 & 0 \\
\hline SEL 01 & Selbach & $28^{\circ} 38^{\prime} 02^{\prime \prime}$ & $52^{\circ} 56^{\prime} 07^{\prime \prime}$ & 0 & 0 \\
\hline TAP 01 & Tapejara & $28^{\circ} 03^{\prime} 44^{\prime \prime}$ & $51^{\circ} 54^{\prime} 58^{\prime \prime}$ & 0 & 0 \\
\hline TAP 02 & Tapejara & $28^{\circ} 04^{\prime} 11^{\prime \prime}$ & $51^{\circ} 58^{\prime} 21^{\prime \prime}$ & 0 & 0 \\
\hline TPE 01 & Tapera & $28^{\circ} 38^{\prime} 44^{\prime \prime}$ & $52^{\circ} 51^{\prime} 14^{\prime \prime}$ & 0 & 0 \\
\hline TAQ 01 & Taquaruçu do Sul & $27^{\circ} 23^{\prime} 56^{\prime \prime}$ & $53^{\circ} 28^{\prime} 45^{\prime \prime}$ & 1 & 0 \\
\hline TAQ 02 & Taquaruçu do Sul & $27^{\circ} 24^{\prime} 01^{\prime \prime}$ & $53^{\circ} 27^{\prime} 54^{\prime \prime}$ & 0 & 0 \\
\hline TPO 01 & Tenente Portela & $27^{\circ} 23^{\prime} 32^{\prime \prime}$ & $53^{\circ} 49^{\prime} 59^{\prime}$ & 1 & 0 \\
\hline TPO 02 & Tenente Portela & $27^{\circ} 23^{\prime} 34^{\prime \prime}$ & $53^{\circ} 47^{\prime} 54^{\prime \prime}$ & 0 & 0 \\
\hline TIH 01 & Tio Hugo & $28^{\circ} 35^{\prime} 28^{\prime \prime}$ & $52^{\circ} 38^{\prime} 02^{\prime \prime}$ & 0 & 0 \\
\hline TIH 02 & Tio Hugo & $28^{\circ} 33^{\prime} 28^{\prime \prime}$ & $52^{\circ} 34^{\prime} 18^{\prime \prime}$ & 0 & 0 \\
\hline TRM 01 & Três de Maio & $27^{\circ} 44^{\prime} 40^{\prime \prime}$ & $54^{\circ} 12^{\prime} 53^{\prime \prime}$ & 0 & 0 \\
\hline TRM 02 & Três de Maio & $27^{\circ} 48^{\prime} 52^{\prime}$ & $54^{\circ} 15^{\prime} 16^{\prime \prime}$ & 0 & 0 \\
\hline TRM 03 & Três de Maio & $27^{\circ} 49^{\prime} 00^{\prime \prime}$ & $54^{\circ} 15^{\prime} 05^{\prime}$ & 0 & 0 \\
\hline TRM 04 & Três de Maio & $27^{\circ} 47^{\prime} 10^{\prime \prime}$ & $54^{\circ} 14^{\prime} 59^{\prime \prime}$ & 0 & 0 \\
\hline TRP 01 & Três Passos & $27^{\circ} 25^{\prime} 33^{\prime \prime}$ & $53^{\circ} 55^{\prime} 22^{\prime \prime}$ & 0 & 0 \\
\hline TRP 02 & Três Passos & $27^{\circ} 29^{\prime} 47^{\prime \prime}$ & $53^{\circ} 53^{\prime} 36^{\prime \prime}$ & 0 & 0 \\
\hline TUC 01 & Tucunduva & $27^{\circ} 36^{\prime} 39^{\prime \prime}$ & $54^{\circ} 28^{\prime} 49^{\prime \prime}$ & 0 & 0 \\
\hline TUC 02 & Tucunduva & $27^{\circ} 39^{\prime} 26^{\prime \prime}$ & $54^{\circ} 27^{\prime} 10^{\prime \prime}$ & 0 & 0 \\
\hline TUC 03 & Tucunduva & $27^{\circ} 36^{\prime} 39^{\prime \prime}$ & $54^{\circ} 28^{\prime} 58^{\prime \prime}$ & 0 & 0 \\
\hline TUP 01 & Tupanciretã & $29^{\circ} 02^{\prime} 59^{\prime \prime}$ & $53^{\circ} 40^{\prime} 55^{\prime \prime}$ & 0 & 0 \\
\hline TUP 02 & Tupanciretã & $29^{\circ} 02^{\prime} 43^{\prime \prime}$ & $53^{\circ} 40^{\prime} 18^{\prime \prime}$ & 0 & 0 \\
\hline TPA 01 & Tuparendi & $27^{\circ} 44^{\prime} 22^{\prime}$ & $54^{\circ} 24^{\prime} 23^{\prime \prime}$ & 0 & 0 \\
\hline VAC 01 & Vacaria & $28^{\circ} 25^{\prime} 25^{\prime \prime}$ & $51^{\circ} 01^{\prime} 19^{\prime \prime}$ & 0 & 0 \\
\hline VAC 02 & Vacaria & $28^{\circ} 30^{\prime} 39^{\prime \prime}$ & $50^{\circ} 51^{\prime} 49^{\prime}$ & 0 & 0 \\
\hline VGR 01 & Victor Graeff & $28^{\circ} 37^{\prime} 52^{\prime}$ & $52^{\circ} 45^{\prime} 32^{\prime}$ & 0 & 0 \\
\hline VLA 01 & Vila Lângaro & $28^{\circ} 08^{\prime} 28^{\prime \prime}$ & $52^{\circ} 12^{\prime} 28^{\prime}$ & 1 & 0 \\
\hline VLA 02 & Vila Lângaro & $28^{\circ} 08^{\prime} 03^{\prime \prime}$ & $52^{\circ} 09^{\prime} 09^{\prime \prime}$ & 0 & 0 \\
\hline VLA 03 & Vila Lângaro & $28^{\circ} 08^{\prime} 34^{\prime \prime}$ & $52^{\circ} 09^{\prime} 08^{\prime \prime}$ & 0 & 0 \\
\hline VLA 04 & Vila Lângaro & $28^{\circ} 08^{\prime} 19^{\prime \prime}$ & $52^{\circ} 09^{\prime} 18^{\prime \prime}$ & 0 & 0 \\
\hline VIA 01 & Vista Alegre & $27^{\circ} 22^{\prime} 35^{\prime \prime}$ & $53^{\circ} 29^{\prime} 34^{\prime \prime}$ & 0 & 0 \\
\hline
\end{tabular}

application criteria (indicated vegetative stage, climate conditions and others) (Gazziero et al., 2009). The use of half the maximum registered dose is justified by the fact that the process of resistance selection may be influenced by the use of sub doses of herbicides and because there is differential susceptibility of biotypes to the application of the herbicide (Neve \& Powles, 2005; Yu et al., 2013; Yu \& Powles, 2014).
The 14 biotypes that survived the application of half the registered dose presented susceptibility difference to the clethodim herbicide, being concentrated in 12 cities of the northwest, northeast and Midwest regions, those being: Água Santa (2), Coqueiros do Sul (2), David Canabarro, Erval Seco, Espumoso, Ibiraiaras, Panambi, Pejuçara, Santo Cristo, Taquaruçu do Sul, Tenente Portela and Vila Lângaro (Figure 2). 
The failures in the control of ryegrass observed in areas with the application of clethodim, in wheat and maize crops in RS, and the result of this study show the control of the biotypes suspected to be resistant, indicating that these failures of control can be a result of other factors. Handling practices such as the use of sub doses of clethodim, intensive use of the herbicide and absence of crop rotation were suggested as causes of failure on the control of Euphorbia heterophylla by glyphosate in RS (Vargas et al., 213), and it can also be the reason for this observation in ryegrass with clethodim herbicide.

The resistance of weeds to herbicides is an evolutionary process and it pre-exists in nature, influenced by the biology of the species and by genetic factors related to the herbicide in question (Maxwell \& Mortimer, 1994) among which the pressure made by the rate of herbicides application is an important factor in the selection of resistant biotypes (Powles $\& \mathrm{Yu}, 2010)$. However, recent studies have shown that recurrent selections with reduced doses (smaller than the registered dose) of ACCase inhibitor herbicides result in a rapid evolution of the resistance to herbicides in only three generations (Neve \& Powles, 2005; Manalil et al., 2011). The susceptibility difference found in the 14 biotypes that

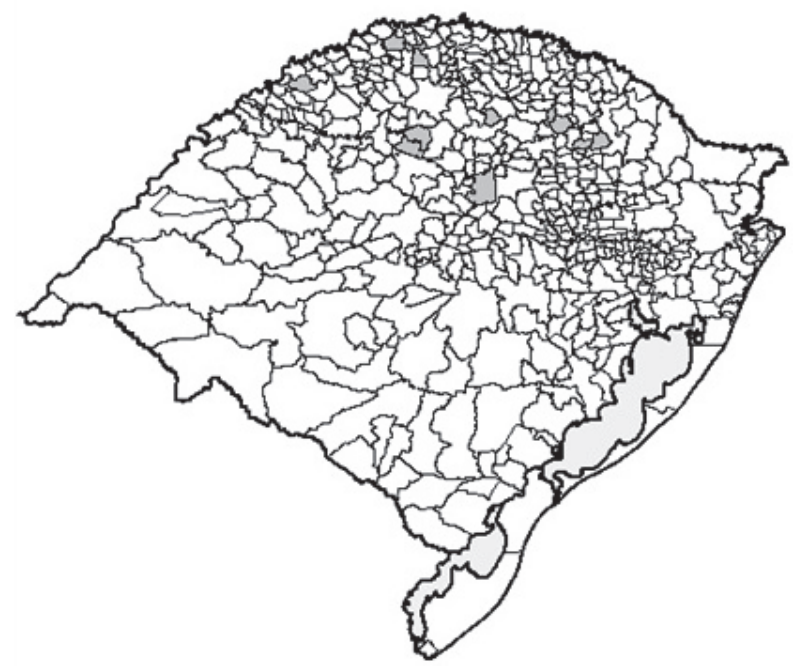

Source: Adapted from Geolivre - Mapas temáticos RS, 2011.

Figure 2 - Geographic location of the cities where there were biotypes with lower susceptibility to the clethodim herbicide in the state of Rio Grande do Sul. survived the application of half the herbicide dose may be a result of the use of low doses, such as 72 at $84 \mathrm{~g}$ i.a. ha ${ }^{-1}$ of clethodim by the producers.

In general, in most cases of resistance to ACCase inhibitor herbicides, the resistance mechanism is related to an alteration in the place of action of the herbicide due to a mutation in the gene that encodes the expression of the ACCase enzyme. However, the metabolization that enables a plant to detoxify the herbicide to non-toxic compounds is also an important mechanism that gives resistance and, in many cases, this mechanism gives crossed or multiple resistance to different herbicides of the chemical group and different action mechanisms (Délye, 2005; Powles \& Yu, 2010). The primary action mode of ACCase inhibitor herbicides consists in the inhibition of the lipid synthesis, because it inhibits the enzyme (Roman, 2007). The ACCase present in poaceae is sensitive to inhibition by these herbicides (Rodrigues, 1994). However, some conditions are recommended so that the herbicides can reach a higher efficiency, such as the application when the plants present an elevated metabolic activity (Pereira et al., 2011).

Thus, according to the results obtained, resistance of Lolium multiforum to clethodim is rejected, but susceptibility difference cannot be discarded. In the field, there are several reports on the failure of ryegrass control with the application of clethodim, which suggests resistance -, a fact that is not confirmed by this paper. The survival of plants in the field occurs without a logical explanation, once it is applied by tractors and with precision sprayer with up to twice the registered dose. The change of environment and the time of growth different from the time when there is chemical control in the field, caused by harvest in the field and sowing in greenhouse, can result in a smaller interference of environmental factors, perhaps interfering in the metabolism of the plants. That way, there is a scientific explanation for the survival of these plants.

On the other hand, one cannot ignore the fact that there are control failures in the field, and that the remaining plants are not 
controlled with doses above the indicated ones in the clethodim label (120 g i.a. ha ${ }^{-1}$ ). Considering that the characteristic is not hereditarily transmitted, the options are reduced to epigenetic factors. Epigenetic resistance happens when a gene is activated and results in the over expression of the target enzyme of the herbicide, or even in the expression of enzymes of the metabolism or kidnapping in the vacuole or intercellular spaces of the herbicide molecule (Gressel, 2009). The epigenetic effects may or may not be transferred to the following generation through meiosis, opposing to the theory of resistance heritability (Bruce et al., 2007).

Considering the results obtained in this paper, it is evident to see the need for an evaluation of epigenetic resistance in ryegrass. In addition, it is worth highlighting the importance of ryegrass handling, especially with the use of integrated handling of weeds, based on the implementation of crop rotation, rotation and/or association of herbicides with different action mechanisms, in order to delay the emergence and evolution of biotypes resistant to herbicides.

Therefore, the biotypes AGS 02, AGS 03, COQ 02, COQ 05, DAC 02, ERS 01, ESP 01, IBR 01, PAN 01, PEJ 03, SCR 01, TAQ 01, TPO 01 and VLA 01 present smaller susceptibility, not being controlled with the application of the maximum registered dose of clethodim for the control of ryegrass.

There is no difference between the sensitive biotypes and resistance suspected that could justify survival, in the field, with the application of $120 \mathrm{~g}$ i.a. ha-1 of clethodim.

\section{ACKNOWLEDGEMENT}

The authors would like to tank CAPES for financing the fellowships of the authors.

\section{LITERATURE CITED}

AGROFIT. Sistema de agrotóxicos fitossanitários. Available at: <http://extranet.agricultura.gov.br/agrofit_cons/ principal_agrofit_cons $>$.Accessed on: 9 jan. 2015.

BRUCE, T. J. A. et al. Stressful "memories" of plants: evidence and possible mechanisms. Plant Sci., v. 173, n. 6, p. 603-608, 2007.

Planta Daninha, Viçosa-MG, v. 34, n. 2, p. 365-373, 2016
CHRISTOFFOLETI, P. J.; LÓPEZ-OVEJERO, R. Principais aspectos da resistência de plantas daninhas ao herbicida glyphosate. Planta Daninha, v. 21, n. 3, p. 507-515, 2003.

DÉLYE, C. Weed resistance to acetyl coenzyme A carboxylase inhibitors: an update. Weed Sci., v. 53, n. 5 , p. 728-746, 2005.

GAZZIERO, D. L. P. et al. Critérios para relatos oficiais estatísticos de biótipos de plantas daninhas resistentes a herbicidas. In: AGOSTINETTO, D.; VARGAS, L. (Ed.) Resistência de plantas daninhas a herbicidas no Brasil. Passo Fundo: Berthier, 2009. p. 91-101.

GRESSEL, J. Envolving understanding of the evolution of herbicide resistance. Pest Manage. Sci., v. 65, n. 11, p. 1164-1173, 2009.

HUGH, J. B. Herbicide-resistant weeds: management tatics and practices. Weed Technol., v. 20, n. 3, p. 793-814, 2006.

LAZAROTO, C. A. et al. Biologia e ecofisiologia de buva (Conyza bonariensis e Conyza canadensis). Ci. Rural, v. 38, n. 3, p. 852-860, 2008.

MANALIL, S. et al. Rapid evolution of herbicide resistance by low herbicides dosages. Weed Sci., v. 59, n. 2, p. 210-217, 2011.

MAXWELL, B. D.; MORTIMER, A. M. Selection for herbicide resistance. In: POWLES, S. B.; HOLTUM, J. A. M. Herbicide resistance in plants: biology and biochemistry. Boca Raton: 1994. p. 1-25.

NEVE, P.; POWLES, S. Recurrent selection with reduced herbicide rates results in the rapid evolution of herbicide resistance in Lolium rigidum. Theor. Appl. Genetics, v. 110, n. 6, p. 1154-1166, 2005.

OWEN, M. J. et al. Multiple herbicide-resistant Lolium rigidum (annual ryegrass) now dominates across the Western Australian grain belt. Weed Res., v. 54, n. 3, p. 314-324, 2014.

PEREIRA, M. R. R. et al. Respostas de plantas de Eleusine indica sob diferentes condições hídricas a herbicidas inibidores da ACCase. Planta Daninha, v. 29, n. 2, p. 397-404, 2011 .

POWLES, S. B.; YU, Q. Evolution in action: plants resistant to herbicides. Ann. Rev. Plant Biol., v. 61, n. 1, p. 317-347, 2010 .

RODRIGUES, J. D. Absorção, translocação e modo de ação de defensivos. Botucatu: UNESP, Instituto de Biociências, 1994. 106 p. 
ROMAN, E. S. Como funcionam os herbicidas: da biologia à aplicação. [S.1]: Berthier, 2007. 160 p.

ULGUIM, A. R. Resposta de capim-pé-de-galinha (Eleusine spp.) ao herbicida glyphosate. 2012. $71 \mathrm{f}$. Dissertação (Mestrado em Agronomia) - Universidade Federal de Pelotas, Pelotas, 2012.

VARGAS, L. et al. Práticas de manejo e a resistência de Euphorbia heterophylla aos inibidores da ALS e tolerância ao glyphosate no Rio Grande do Sul. Planta Daninha, v. 31, n. 2, p. 427-432, 2013.
VIDAL, R. A.; MERROTO JR., A. Herbicidologia. Porto Alegre: Evangraf, 2001. 152 p.

YU, Q. et al. Enhanced rates of herbicide metabolism in low herbicide-dose selected resistant Lolium rigidum. Plant Cell Environ., v. 36, n. 4, p. 818-827, 2013.

YU, Q.; POWLES, S. B. Metabolism-based herbicide resistance and cross-resistance in crop weeds: a threat to herbicide sustainability and global crop production. Plant Physiol., v. 166, n. 3, p. 1106-1118, 2014. 